\title{
Ideograms for Physics and Chemistry
}

\author{
Pablo García Risueñd* \\ Institut für Physikalische Chemie / Center for Ultrafast Imaging Universität Hamburg, \\ Grindelallee 117, 20146 Hamburg, Germany \\ Apostolos Syropoulos \\ Greek TeX Friends, 28 Oktovriou 366A, GR-67133 Xanthi, Greece \\ Natàlia Vergés \\ Ars Didactica, C/ Major 2, 17850 Besalú, Girona, Spain
}

(Dated: October 11, 2016) 


\begin{abstract}
Ideograms (symbols that represent a word or idea) have great communicative value. They refer to concepts in a simple manner, easing the understanding of related ideas. Moreover, ideograms can simplify the often cumbersome notation used in the fields of Physics and physical Chemistry. Nonetheless only a few specific ideograms for these fields - like $\hbar$ and $\AA$ - have been defined to date. In this work we propose that the scientific community follows the example of Mathematics -as well as that of oriental languages- and bestows a more important role upon ideograms. To support this thesis we propose ideograms for essential concepts in Physics and Chemistry. They are designed to be intuitive, and their goal is to make equations easier to read and understand. Our symbols are included in a publicly available LTEXpackage (svrsymbols).
\end{abstract}

Keywords: Communication of science / Clear notation / Ideograms / Understanding of Physics / Psychology of science / Elementary particles / Error / Assumption

PACS: $01.20 .+\mathrm{x}, 01.10 .-\mathrm{m}, 01.90 .+\mathrm{g}$

The final publication is available at link.springer.com, DOI 10.1007/s10701-016-0044-5. 


\section{INTRODUCTION}

Ideograms are present in the communication codes that we use daily. All around the world, different kinds of ideograms are ubiquitous in daily life. Well known examples are traffic signs, musical notes, and commercial logos. In written text, remarkable examples of ideograms are the integer signs from 0 to 9 and other mathematical symbols, like $+,-, \%, \cdot \times, \sqrt{ }, \in$. Symbols that represent a word, like @, $\$$ or $\&$, are common in texts written in English. Ideograms are still more important in oriental languages: Japanese and Korean use mixed writing systems, with both phonetic (syllabic) and ideographic signs; in Japanese, almost all written sentences contain characters of both types, while Chinese, the language of more than one billion people, is mainly ideographic.

For people whose mother tongue uses alphabetical and ideographic writing, ideograms convey a better and faster comprehension than the corresponding words written in phonetic

characters [1]. Moreover, concepts represented with ideograms are better identified and recalled [2]. When reading a word written in alphabetical (or syllabic) characters, it is first necessary to identify every individual character (letter), then give them a phonetical value, and finally to associate the sound with the concept; in contrast, ideographic writing is simpler. It avoids the intermediate step, and so the meaning of the character (ideogram) can be directly understood. One can intuitively gauge the advantage of ideograms by merely comparing the ease of reading numbers $(1,2,3 \ldots)$ and their written names (one, two, three ...); this phenomenon was thoroughly studied in [3]. The expressive power of ideograms is also illustrated by the dramatic fact that there exist people with neurological injuries who cannot read texts written with phonetic signs, but they can read and understand well if the text is written with ideograms [4].

Signs are often classified into symbols, indices or icons depending on its similarity with the object they refer to. Symbols are purely abstract, while indices are somewhat connected to the object, and icons resemble the object. The proximity of signs and their signified objects helps to recall the latter in a more vivid way. Umberto Eco, a celebrated expert in semiotics, stated that an icon can acquire primacy over its signified due to familiarity: "At a certain point the iconic representation, however stylized it may be, appears to be more true 
than the real experience, and people begin to look at things through the glasses of iconic convention" [5]. This statement is exemplified e.g. by experiments that prove that human mind identifies the concept 'hand' on a cartoon of it more quickly than on a photography of a hand [6]. Albert Einstein, frequently considered the best scientist ever, stated that he did not think in terms of words, but of symbols [7].

We propose that the power of ideograms should be more often utilized by the scientific community. The fact that this community has been mostly composed by westerners has probably precluded this possibility so far. However, the current complexity of equations suggests a universal notation which is as intuitive and univocal as possible. Despite the close relationship between Mathematics and Physics, ideograms are ubiquitous in Mathematics, but shyly used in Physics. Examples of used ideograms are $\hbar$ (reduced Planck constant), $\AA$ (angstrom) or symbols for particles used in particle Physics (see appendix). We wish to advocate for the definition of new ideograms for the most important concepts of Physics and Chemistry. The form and the meaning of such ideograms should be agreed by the community, e.g. in specialized conferences as those dedicated to the definition of units [8]. These ideograms should be simple and intuitive, and they should clearly point to specific concepts to avoid any confusion to their meaning.

In order to boost the trend of using ideograms in Science, we present below examples for the representation of basic concepts of Physics and Chemistry. All symbols presented out of the appendix are original, except those of atom, spin, hole and the first symbols for particles on table I (the appendix also contains symbols traditionally used in particle Physics). We welcome suggestions for additional new symbols in any field of Physics and Chemistry.

We have tried to design ideograms which are intuitive and easy to remember. To this end we based many of our designs on letters of the Latin alphabet and we tried to give them an iconic or index-character. For example, we added wiggly lines at the ideograms for 'photon' or 'phonon', and the symbol for 'graphene' is nearly an hexagon. To ease their identification, some of our designs contain the initial letter of the word that they represent. The chosen font in most cases is cmmi12 or cmr10, which are widely used for writing equations. 


\section{IDEOGRAMS FOR GENERAL CONCEPTS}

The six signs on table $\llbracket$ are generic, they can be used in any discipline.

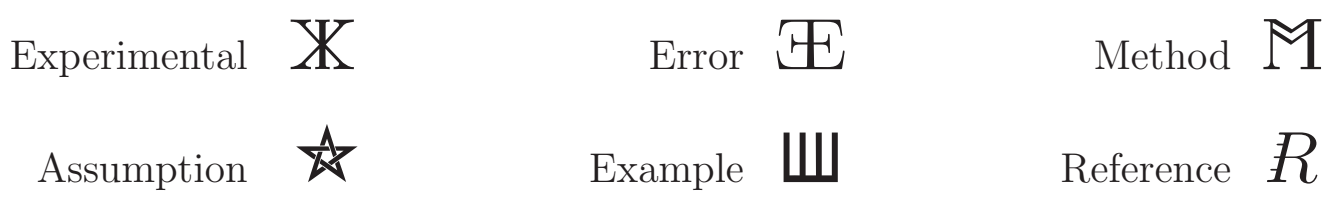

TABLE I: Ideograms for generic concepts.

The use of $\mathbb{E}$ makes it clear that the represented quantity means 'error'; the use of $E$ or $e$ instead would conflict with 'energy', '2.71828..', 'charge of the electron', etc. 'Err' has three letters which makes it slower to read and could be misinterpreted as the product of three quantities. The use of $\mathbb{X}$ instead of 'exp' can avoid the confusion with 'exponential'. Naturally every scientist does understand the concepts behind traditional notation (like 'Err' or 'exp'); but the need of making a short-time reflection on the meaning of these characters distracts from the main goal of understanding the physical meaning of the equation in question. Note that $\mathbb{X}$ is different to the Cyrillic character 'Zhe'.

The sign of assumption ( ) was chosen to make it stand out when browsing a page. Frequently, is difficult to locate the underlying assumptions in many scientific papers and a scientist may spend an inordinate amount of time before discovering that the paper is not relevant for his own line of investigation. If a big $\star$ sign appears whenever an assumption is made, the reader will immediately be able to determine if the paper is suitable for his

research. We recommend the use of the notation $\star \star 1, \star 2, \ldots, \star n$ for the 1 st, 2 nd, . , $n$-th assumptions, for the sake of future references. 


\section{IDEOGRAMS FOR PARTICLES AND QUASIPARTICLES}

The signs in the first two rows of table (among others) are used in particle Physics, but to the best of our knowledge they have never included into a $\mathrm{AT}_{\mathrm{E} X} \mathrm{X}$ package. We designed them to be more elegant than their math mode counterparts $\left(e^{-}, p^{+}, \ldots\right)$. The existence of ideograms for particles like 'electron' makes it advisable to have at our disposal specific signs for other widely used concepts in Physics, such as 'photon' or 'phonon'.

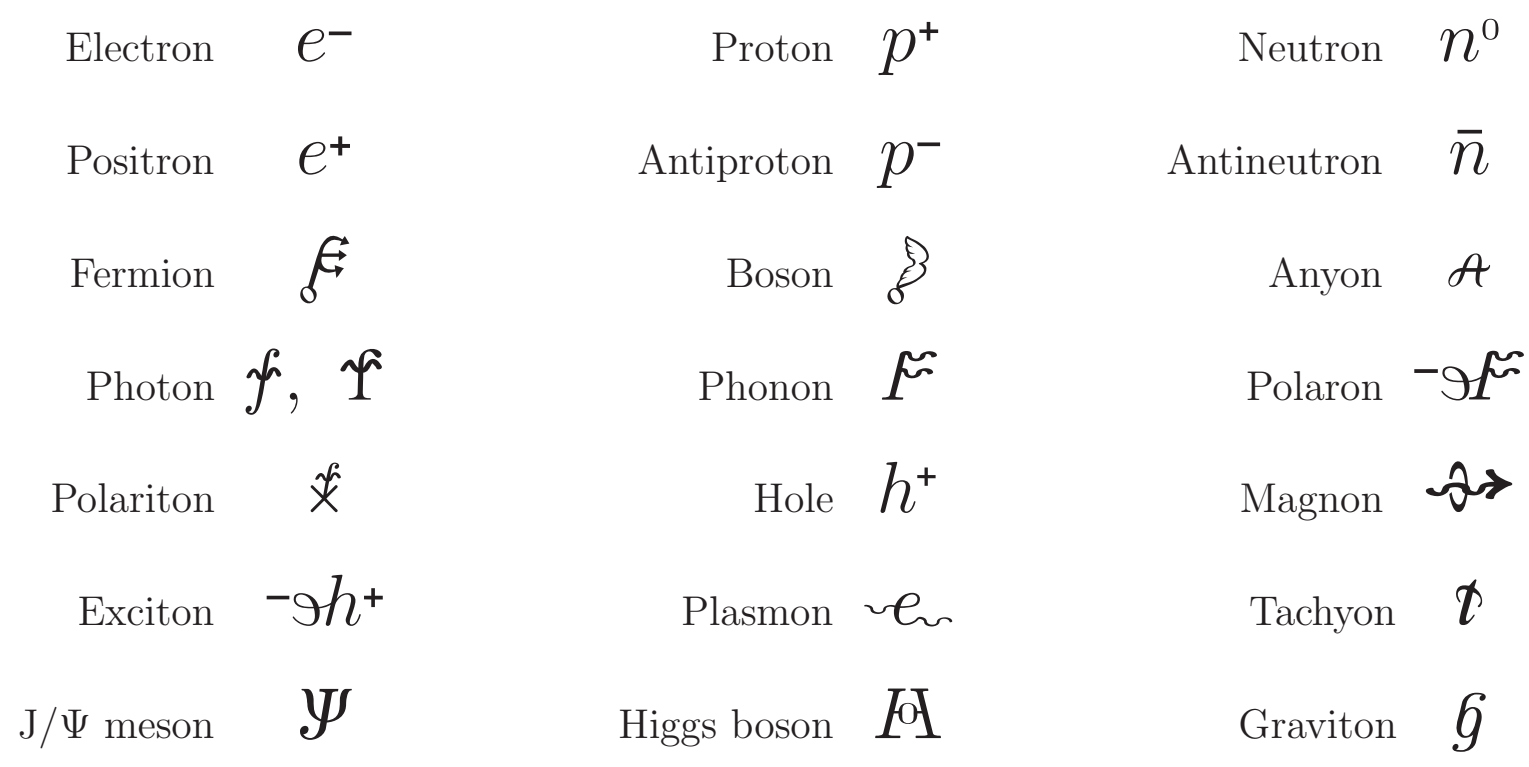

TABLE II: Ideograms for particles and quasiparticles.

The wiggly lines in the ideograms of table $\amalg$ emphasize on the wave nature of (quasi)particles. The signs of 'photon' and 'phonon' are based on the ' $f$ ' and ' $F$ ' characters due to the equivalence between the 'ph' and 'f' sounds. The symbol of 'magnon' is similar to that of 'spin' (see table [V]), but suggesting a wave propagation. The ideogram for 'graviton' resembles a spiral galaxy. The sign of ' $x$ ' within the 'polariton' represents an excitation in the material. The symbols of boson and fermion require some further explanation. During the middle ages, a typical topic of philosophical discussion was the question: "How many angels can stand on the point of a pin?". The accepted answer was "An infinity" due to the immaterial character of angels. The symbol of 'boson' resembles a $B$ formed with the wings of an angel (because bosons, like angels, do not obstruct each other). In contrast, the 
symbol of 'fermion' resembles an $F$ formed with the trident of a demon (designing atomic weapons as F. did is considered by many to be somewhat devilish).

We also include many symbols that are used at the field of particle Physics into the svrsymbols package. They can be viewed in the appendix.

\section{IDEOGRAMS FOR CONCEPTS OF CHEMISTRY}

The symbols presented on table III are a suggestion of some ideograms for basic concepts of Chemistry. We suggest the design of many others, pointed by the Chemistry community.

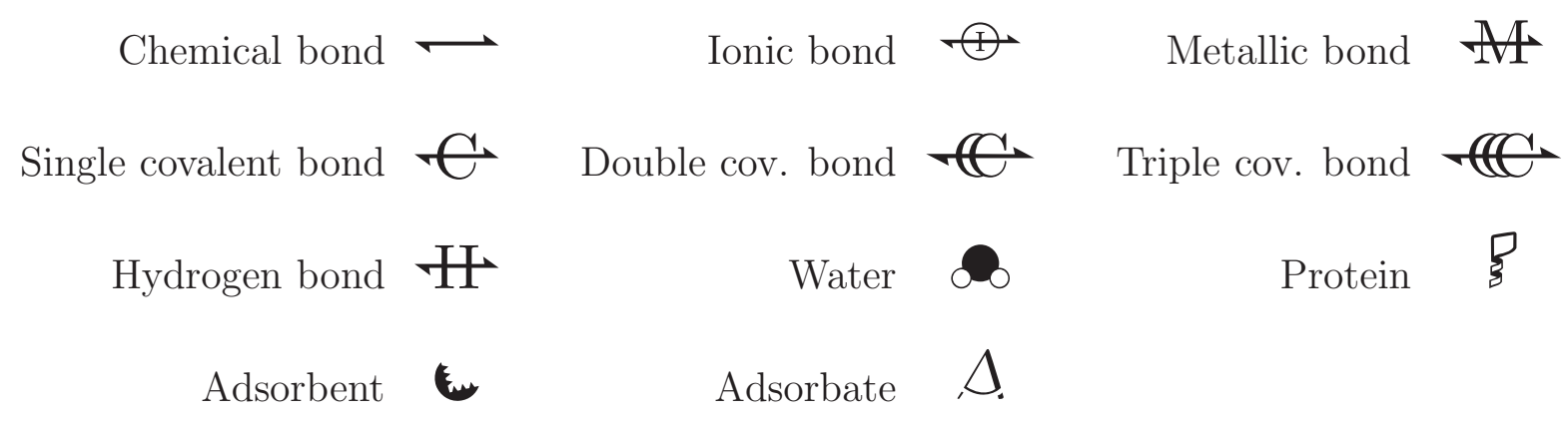

TABLE III: Ideograms for basic concepts of Chemistry.

The signs for bonds on table \II have the shape of a double harpoon to stress the nature of bonds as joining objects. The different kinds of bonds are specified by their initials to ease their identification. The ideogram for 'protein' is a P-shaped alpha-helix, while the 'adsorbate' is a modified A and the sign of 'adsorbent' emphasizes on its capability to join to the adsorbate. 


\section{IDEOGRAMS FOR OTHER SYSTEMS AND PHENOMENA}

The symbols presented on table IV correspond to a variety of concepts which are commonly used in Physics.

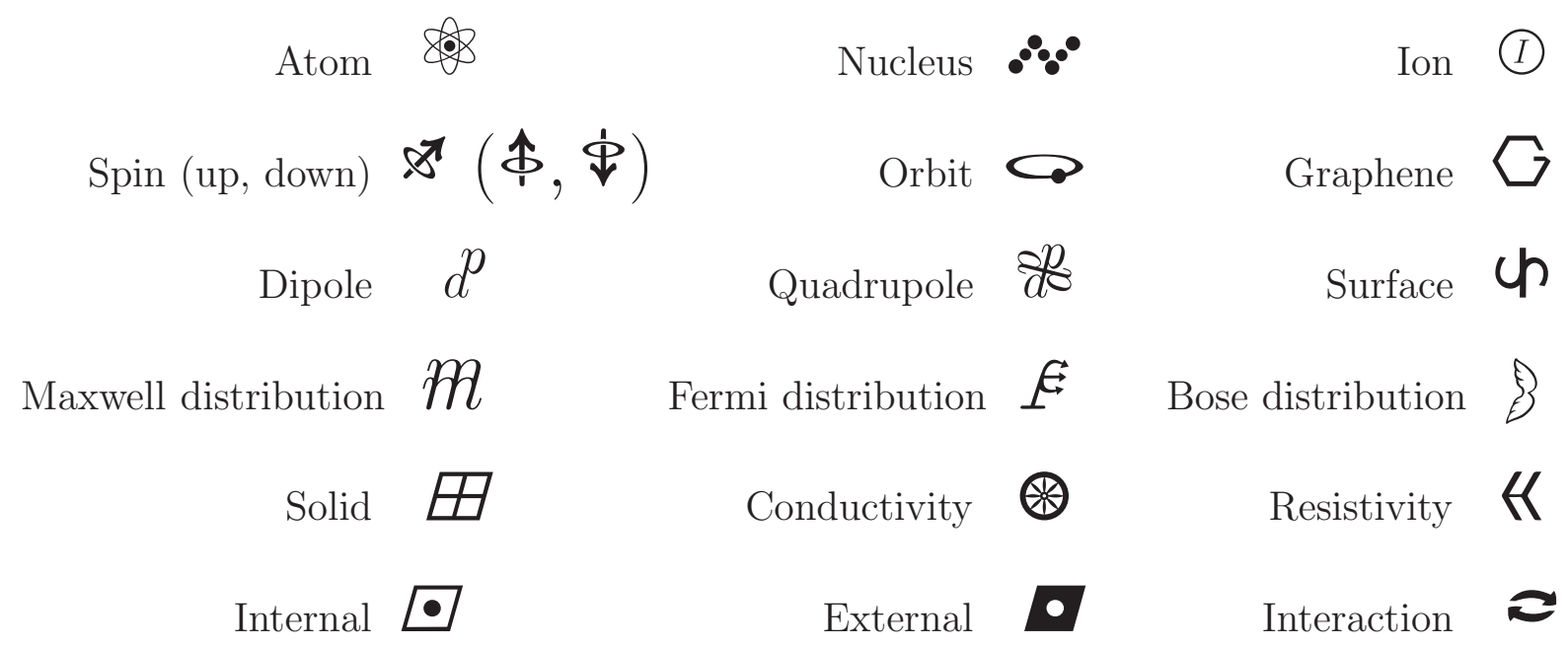

TABLE IV: Ideograms for several concepts of Physics.

The ideogram of 'dipole' on table IV is made of a $d$ and a $p$ resembling two charged spheres at the terminals of a segment; the ideogram of 'quadrupole' is made of two symbols of 'dipole'. The symbol of 'surface' is based on an S, while that of 'graphene' is based on a G (with a hexagonal shape, like the primitive cell of graphene). The symbol of conductivity is wheel-shaped to stress on the mobility of charges, and the ideogram of 'resistivity' tries to hint the obstruction to the motion.

Let us see some examples on how the proposed ideograms simplify the notation. A typical expression for a Hamiltonian depending on electronic and phononic parts is $H=H_{e l}+$ $H_{p h}+H_{e l-p h}$. With our simplified notation, its expression would be $H=H_{e^{-}}+H_{\digamma^{-}}+H_{e^{-} \digamma^{*}}$, or $H=H_{e^{-}}+H_{f^{*}}+H_{\mathbf{s}}$. The example of a table where the results and errors of different calculation methods are compared with experimental results looks as follows: 


\begin{tabular}{|c|c|c|c|c|}
\hline Method 1 & Err.meth. 1 & Method 2 & Err.meth. 2 & Exp. \\
\hline \hline 1.01 & 0.12 & 1.12 & 0.03 & 1.15 \\
2.56 & 0.14 & 2.28 & 0.02 & 2.24 \\
\hline
\end{tabular}

\begin{tabular}{|c|c|c|c|c|}
\hline M1 & $\mathbb{E}_{\mathrm{M} 1}$ & M2 & $\mathbb{E}_{\mathrm{M} 2}$ & $\mathbb{X}$ \\
\hline \hline 1.01 & 0.12 & 1.12 & 0.03 & 1.15 \\
2.56 & 0.14 & 2.28 & 0.02 & 2.24 \\
\hline
\end{tabular}

TABLE V: Comparison of data presentation without and with the use of ideograms.

In these examples it is clear that the ideographic notation is simpler, and it does not require the reader to decode the phonetical value and the meaning of a set of letters because the meaning of an ideogram is univocal. Such examples illustrate the usefulness and the communication capabilities of ideograms in Physics and Chemistry.

We expect that the presented ideograms are themselves useful for the scientific community, and that they promote the use of specifically-devised ideograms for basic concepts of Physics and Chemistry, as it is customary and useful in both oriental languages and Mathematics.

\section{ACKNOWLEDGMENTS}

We are very grateful to Carl Christian K. Mikkelsen (University of Umeå, Sweden) for his help and advice.

\section{AUTHOR CONTRIBUTIONS}

P.G.R. proposed the idea of developing and using further ideograms and made by hand the corresponding designs; both N.V. and A.S. worked as graphical designers; A.S. wrote the $\mathrm{HT}_{\mathrm{E} X}$ package that includes the ghlyphs. 


\section{Appendix: Table of glyphs of the svrsymbols package}

The SVRsymbols font contains new ideograms for use in Science. It also contains a standard presentation for ideograms which are traditionally used in particle Physics (e.g. $\left.e^{-}, p^{+}\right)$but which have not previously been included in $\mathrm{AT}_{\mathrm{EX}}$. We included them into the svrsymbols package so that there is a standard presentation that boosts the use of ideograms in Science. For some concepts there existed a sign but we propose an alternative ideogram to simplify the notation $(J / \Psi \rightarrow \Psi)$ or to avoid confusion $\left(\right.$ e.g. $\gamma \rightarrow \mathcal{f} ; H^{0} \rightarrow F$; $\left.G \rightarrow g\right)[9]$. We suggest to gradually change the rest of characters used for particles (e.g. $c, d, g$ ) by specifically-devised ideograms. The novel symbols have been designed to be intuitive and easy to identify and to remember. The SVRsymbols package has no options and it provides an interface to the font. It defines commands for use in math mode. The commands and the resulting symbols are shown in the table below. The package can be downloaded from http://ctan.org/pkg/svrsymbols and freely used if the present letter is cited.

\begin{tabular}{|c|c|c|}
\hline Adsorbent (\adsorbent) & (2) & Adsorbate (\adsorbate) \\
\hline Antimuon (\antimuon) & $\mu^{+}$ & Antineutrino (\antineutrino) \\
\hline Antineutron (\antineutron) & $\bar{n}$ & Antiproton (\antiproton) \\
\hline Antiquark (\antiquark) & $\bar{q}$ & Anyon (\anyon) \\
\hline Assumption (\assumption) & & Assumption (\bigassumption) \\
\hline Assumption ( \biggassumption) & & Assumption (\Bigassumption) \\
\hline Atom (\atom) & & $B^{+}$meson ( $\backslash$ Bmesonplus $)$ \\
\hline$B^{0}$ meson $(\backslash$ Bmesonnull $)$ & $B$ & $B^{-}$meson ( $\backslash$ Bmesonminus $)$ \\
\hline Bose distribution ( \boseDistrib) & 8 & Boson ( \boson) \\
\hline Bottom antiquark (\antiquarkb) & $\bar{b}$ & Bottom quark (\quarkb) \\
\hline
\end{tabular}


Charm antiquark (\antiquarkc) $\bar{C}$
Chemical bond (\bond) $D^{+}$meson $(\backslash$ Dmesonplus $) \quad D^{+}$ $D^{-}$meson (\Dmesonminus) $D^{-}$

Dbl. cov. bond (\doublecovbond) Down quark (\quarkd) d Error (\errorsym) 尼 Exciton (\exciton) $-\boldsymbol{g h}^{+}$

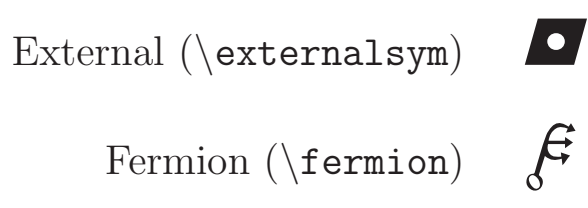
Graphene (\graphene) Higgs boson (\Higgsboson) FI Hydrogen bond (\hbond) $\quad$ I Internal (\internalsym) $\bullet$ Ionic bond (\ionicbond) Kaons (\Kaonplus) $D^{+}$
Kaons (\Kaonminus) $D^{-}$ Maxwell distrib. (\maxwellDistrib) $\overparen{A}$ Method (\method) M
Charm quark (\quarkc) C Conductivity (\conductivity) $D^{0}$ meson $(\backslash$ Dmesonnull $) \quad D^{0}$ Dipole $(\backslash$ dipole $) \quad d$ Down antiquark (\antiquarkd) $\bar{d}$ Electron (\electron) $\quad e^{-}$ Example (\svrexample) Ш Experimental (\experimentalsym) X Fermi distribution $(\backslash$ fermiDistrib) Gluon (\Gluon) $g$ Graviton (\Graviton) $\quad G$

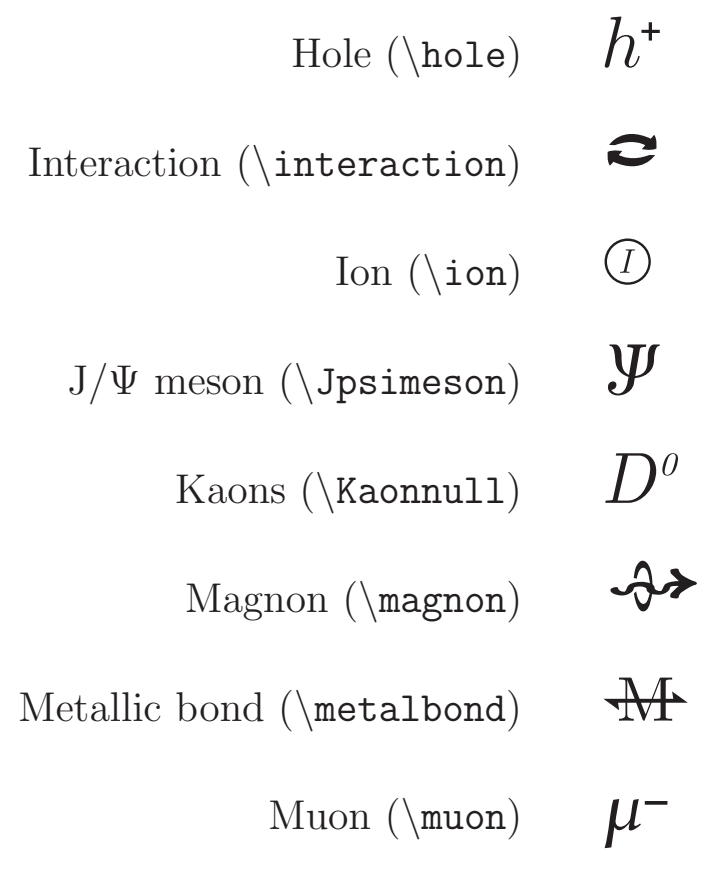


Neutrino (\neutrino) $\quad \boldsymbol{V}$

$$
\text { Nucleus (\nucleus) }
$$

Phi mesons (\phimeson)

Phonon (\phonon) F

Pion plus (\pionplus) $\pi^{+}$

Pion minus (\pionminus $) \quad \pi^{-}$

$$
\text { Polariton (\polariton) } \notin
$$

Positron (\positron) $\quad e^{+}$

Proton (\proton) $\quad p^{+}$

$$
\text { Quark (\quark) } q
$$

Resistivity (\resistivity)

Rho meson 0 (\rhomesonnull)

Single covalent bond (\covbond)

$$
\begin{aligned}
& \text { Spin }(\backslash \operatorname{spin}) \quad \mathscr{T} \\
& \text { Spin up (\spinup) } \\
& \text { Strange quark (\quarks) } S \\
& T^{+} \text {meson (\Tmesonplus) } T^{+} \\
& T^{-} \text {meson (\Tmesonminus) } T^{-}
\end{aligned}
$$

Neutron (\neutron) $\quad n^{0}$

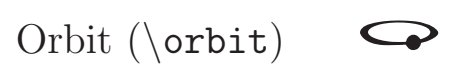

Phi mesons (\phimesonnull) $\quad \phi^{0}$ Photon (\svrphoton, \varphoton) $f, \uparrow$

Pion 0 (\pionnnull) $\pi^{0}$

Plasmon (\plasmon) ver

Polaron (\polaron) -

Protein (\protein)

Quadrupole (\quadrupole) $\not g$

Reference $(\backslash$ reference $) \quad R$

Rho meson plus (\rhomesonplus) $\varrho^{+}$

Rho meson minus (\rhomesonminus) $\varrho^{-}$

Solid (\solid) $\#$

Spin down (\spindown $) \$$

Strange antiquark (\antiquarks) $\quad \bar{S}$

Surface $(\backslash$ surface) फ

$T^{0}$ meson $(\backslash$ Tmesonnull $) \quad T^{0}$

Tachyon (\tachyon) $\not$

Tau lepton plus (\tauleptonplus) $\quad \tau^{+}$Tau lepton minus (\tauleptonminus) $\boldsymbol{\tau}^{-}$ 


$$
\text { Top antiquark (\antiquarkt) } \quad \bar{t} \quad \text { Top quark (\quarkt) } t
$$

Triple cov. bond (\triplecovbond) $\quad$ Up antiquark (\antiquarku) $\quad \bar{u}$

$$
\begin{aligned}
& \text { Up quark (\quarku) } \quad u \quad \text { Upsilon meson (\Upsilonmeson }) \quad \mathcal{Y} \\
& \text { Water (\water) W W bosons (\Wboson) } W \\
& \text { W bosons (\Wbosonplus) } \quad W^{+} \quad \text { W bosons (\Wbosonminus) } \quad W^{-} \\
& \text {Z boson ( } \backslash \text { Zboson }) \quad Z
\end{aligned}
$$

TABLE VI: Ideograms of the svrsymbols ${ }^{A} T_{E} X p a c k a g e$ with their corresponding commands.

* For comments on conceptual issues and suggestions of new ideograms: pablo.risueno@chemie.uni-hamburg.de

$\dagger$ For technical respects: asyropoulos@yahoo.com

[1] A. P. Shinamura, The American Journal of Psychology 100, 15 (1987).

[2] S. Park and T. Y. Arbuckle, Journal of Experimental Psychology: Human Learning and Memory 3(6), 631 (1977).

[3] D. Besner and M. Coltheart, Neuropsychologia 17, 467 (1979).

[4] S. Sasanuma, Brain and Language 2, 369 (1975).

[5] U. Eco, A Theory of Semiotics (Indiana University Press, Bloomington, IN, 1976).

[6] T. A. Ryan and C. B. Schwartz, American Journal of Psychology 96, 66 (1956).

[7] H. E. Gruber and K. Bödeker, eds., Creativity, Psichology and the History of Science (Springer, 2005).

[8] E.g. the General Conference on Weights and Measures, see www.bipm.org, www. bipm.org/utils/common/pdf/CGPM/CGPM25.pdf.

[9] Variable $\gamma$ is frequently a summation index which can represent a wide variety of variables; $H^{0}$ may be misunderstood as a Hamiltonian; $G$ can represent the gravity constant, among many other quantities whose names begin with a 'G'. 